\title{
过渡金属催化的偶联反应在合成 $C$-糖苷中的应用
}

\author{
廖进喜刘慧孙建松* \\ (江西师范大学国家单糖化学合成工程技术研究中心 南昌 330022)
}

\begin{abstract}
摘要 $C$-糖苗是指糖苷键的环外氧原子被碳所取代的一类化合物的总称, 其结构单元广泛存在于天然产物和药物分子 中. 与相应的 $O$-糖苷和 $N$-糖苷相比, $C$-糖苷具有更好的酶稳定性以及耐水解性能. 由于其独特的化学结构和广泛的应 用价值, 其合成方法的研究已经成为当今糖化学领域的一个研究热点. 主要对过渡金属催化的偶联反应构建 $C$-糖苷的 方法进行综述, 试图归纳总结出不同合成方法的特点以及不足, 为过渡金属催化 $C$-糖苷的进一步合成研究提供帮助. 关键词 $C$-糖苷; 合成; 过渡金属催化; 偶联反应
\end{abstract}

\section{Applications of Transition Metal Catalyzed Coupling Reactions in the Synthesis of $\boldsymbol{C}$-Glycosides}

\author{
Liao, Jinxi Liu, Hui Sun, Jiansong* \\ (National Engineering Research Center for Carbohydrate Synthesis, Jianxi Normal University, Nanchang, 330022)
}

\begin{abstract}
C$-Glycosides in which the interglycosidic oxygen atom have been replaced by carbon atom are widely found in natural products and drug molecules. They have better enzymatic and hydrolytic stablility compared to their corresponding $O$-glycosides and $\mathrm{N}$-glycosides. The syntheses of them have received considerable attention because of their unique chemical structure and extensive application value. Rencent advances in the synthesis of $C$-glycosides through coupling reactions catalyzed by transition metal are summarized in this review. A summary of the advantages and disadavatages of different synthetic methods will be beneficial to the develepment of new synthetic method for $C$-glycosides.
\end{abstract}

Keywords $C$-glycosides; synthesis; transition metal catalyzed; coupling reactions

糖，又称碳水化合物，与蛋白质、核酸和脂质一起 并称为生命活动过程中的四类重要的生物大分子. 它广 泛分布于自然界中, 不仅以葡萄糖、果糖、核糖、蔗糖、 麦芽糖、几丁质、淀粉和纤维素等还原糖的形式存在, 还 与其它非糖分子通过共价键连接形成糖缀合物.

所谓 $C$-糖苷是指糖苷键的环外氧原子被碳所取代 的一类化合物的总称. 这类化合物的合成在近年来受到 了越来越多的研究人员的重视, 究其原因, 一方面是因 为 $C$-糖苷与其具有类似结构的 $O$-糖苷和 $N$-糖苷相比, 具有更好的酶稳定性 ${ }^{[1]}$ 及耐水解性能 ${ }^{[2]}$; 而另一方面则 是因为越来越多的含 $C$-糖苷键的天然产物的发现 ${ }^{[3]}$, 例 如海葵毒素 ${ }^{[4]}$ 等, 使人们迫切地需要寻找到更高效的合 成 $C$-糖苷键的方法.

迄今为止, 已有许多关于 $C$-糖苷的合成方法的报
道 ${ }^{[5]}$, 主要包括过渡金属催化的偶联反应、亲核取代、 重排反应和自由基反应等. 但是鉴于过渡金属催化的 $\mathrm{C}-\mathrm{C}$ 键的偶联反应已经成为有机合成中构建 $\mathrm{C}-\mathrm{C}$ 键 的强有力的工具 ${ }^{[6]}$, 但是在糖化学中的应用却还不是很 普遍，因此本文主要针对过渡金属催化的偶联反应合成 $C$-糖苷的研究方法进行综述, 试图根据不同的过渡金属 参与的 $C$-糖苷的合成研究现状来归纳总结出各类过渡 金属催化剂的特点以及不足, 为过渡金属催化的偶联反 应制备 $C$-糖苷的进一步研究提供帮助.

\section{Pd 催化}

\section{$1.1 \mathrm{Pd}$ 催化的糖烯参与的偶联反应制备 C-苷}

1976 年, Bergstrom 等 ${ }^{[7]}$ 发现嘧啶的录盐与烯烃能够 在 $\operatorname{Pd}(\mathrm{II})$ 的催化下发生偶联反应, Daves 等 ${ }^{[8]}$ 据此推测,

* Corresponding author. E-mail: jssun@jxnu.edu.cn

Received December 8, 2016; revised February 13, 2017; published online February 27, 2017.

Project supported by the National Natural Science Foundation of China (No. 21202166) and the Science and Technology Project Founded by the Education Department of Jiangxi Province (No. GJJ150328).

国家自然科学基金(No. 21202166)和江西省教育厅科学技术研究(No. GJJ150328)资助项目. 
与烯烃具有相似化学性质的糖烯应该也能够在这一条 件下与嘧啶录盐发生偶联反应生成碳核苷类化合物. 经 过试验, 他们发现 3,4-二氢吡喃 1 与嘧啶的醋酸录盐 4 在 1 equiv. 醋酸钯和 2 equiv. 氯化锂的存在下, 得到双键 转移的偶联产物 $5(66 \%)$ 和 $6(24 \%)$. 当采用 3,4-二- $O$-乙 酰基- $D$-阿拉伯糖烯时, 却只以 20\%的收率得到 3-脱乙 酰基的偶联产物 7 (20\%), 另外还得到了部分开环产物 8 $(32 \%)$, 而当采用 $3,4,6-$ 三- $O$-乙酰基葡萄糖烯时，同样 也得到了少量的偶联产物 $9(20 \%)$ 和大部分的开环产物 $10(73 \%)$ (Eq. 1).

作者通过对反应机理的研究, 发现造成各偶联产物 不同的原因在于 $\mathrm{Pd}$ 加成产物的还原脱除步骤 ${ }^{[9]}$ (Scheme 1). 嘧啶的醋酸录盐先与醋酸钯发生金属转移反应, 接 着该嘧啶金属试剂再与糖烯 3 的双键发生加成反应. 加 成会发生在空间位阻相对更小的 $\alpha$ 面得到加成产物 14(或 15), 接下来该加成产物不同的还原脱除路径便会
导致不同的偶联产物的生成.

上述产物中各组分的比例除了底物本身的影响外, 与反应体系的组成特别是反应中所添加的盐的性质密 切相关. 而反应的立体选择性则取决于分子中各个羟基 保护基的大小和化学性质，这一特性在呋喃型糖烯与嘧 啶录盐在 $\mathrm{Pd}$ 催化下进行偶联时表现相当明显 ${ }^{[10]}$ (Eq. 2). 例如当 $\alpha, \beta$ 面空间位阻差不多时(如 16a), 得到的是 $\alpha, \beta$ 混合物(17a, 18a), 而当 $\beta$ 面有大位阻的 TBDPS 保护时 (16b), 只得到了 $\alpha$ 型产物 $\mathbf{1 7 b}$.

2001 年, Maddaford 等 ${ }^{[11]}$ 发现全乙酰化的葡萄糖烯 也能够与芳基嗍酸在 $\mathrm{Pd}$ 的催化作用下发生偶联反应, 生成 2,3-二脱氧的芳基碳苷，但是反应产物并不是他们 所预期的 $\beta-\mathrm{H}$ 消除的产物, 而是类 Ferrier 重排类型的产 物(Eq. 3). 作者推测此反应发生的主要原因是由于在这 一条件下，反式消除要优先于顺式的 Heck- $\beta$-H 消除 ${ }^{[12]}$. 他们也曾试图通过改变反应体系的酸碱性及反应温度,<smiles>[R1]C1C=CC2[R]C1C2[R]</smiles><smiles>Cn1cc(OC(=O)O[Na])c(=O)n(C)c1=O</smiles><smiles>[R]C([R])/C=C\[PH]</smiles><smiles>[R]/C(=C\[3H])C([R])C([R])O</smiles><smiles>[Y8]C1[CH+]C2CCC(C1)OC2</smiles>

1: $R^{1}=R^{2}=R^{3}=R^{4}=H$ 2: $R^{1}=R^{3}=H, R^{2}=R^{4}=O A C$
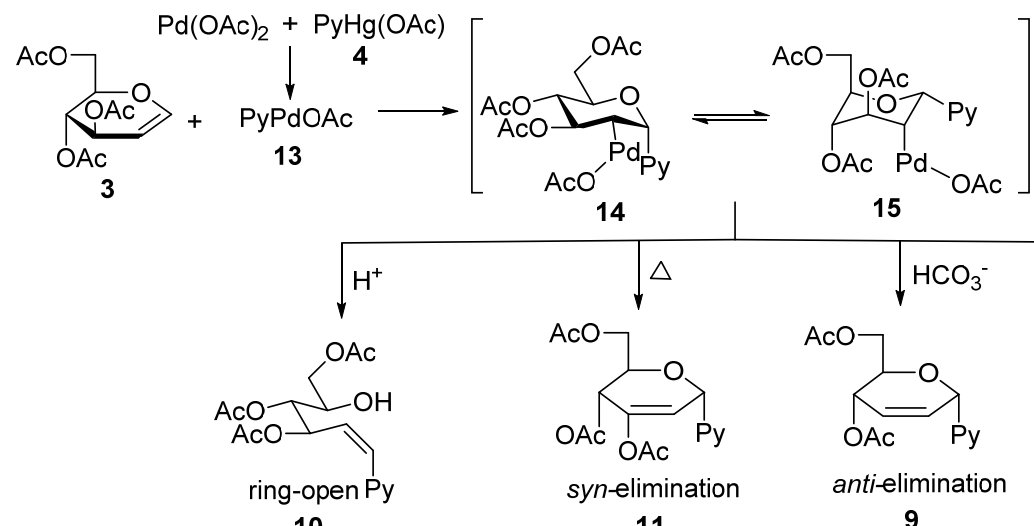

10

11
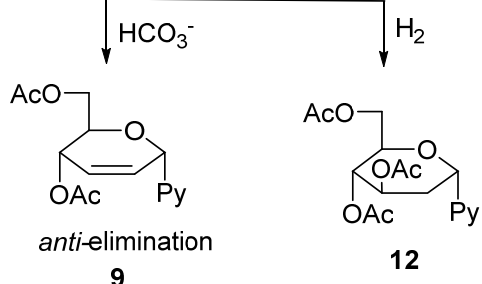

12

图式 $1 \mathrm{Pd}$ 催化的糖烯与嘧啶录盐的偶联反应机理

Scheme 1 Proposed mechanism of $C$-glycoside formation by coupling of glycals and oragnomercury compounds catalyzed by Pd

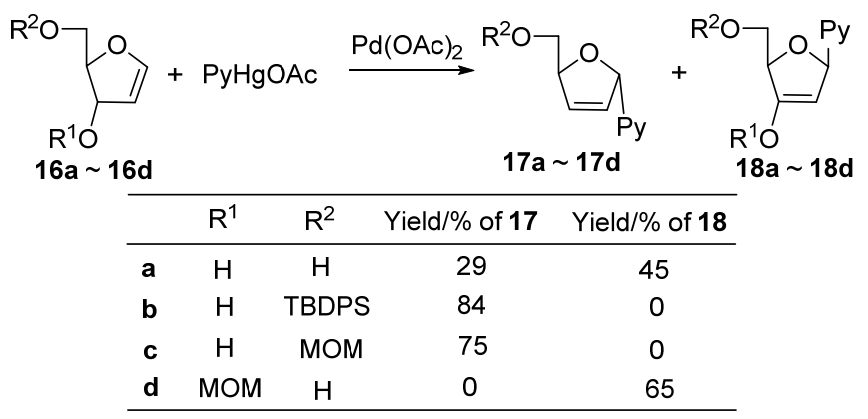




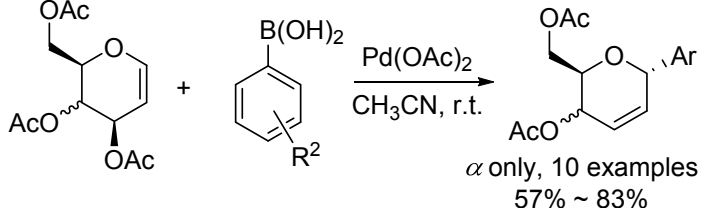

以期能够得到正常的 $\beta-\mathrm{H}$ 消除的产物，但是都没有成功.

2009 年, $\mathrm{Ye}$ 等 ${ }^{[13]}$ 发现糖烯与芳基嗍酸在氧气和醋 酸铜的存在下, 通过醋酸钯催化, 能生成对应的 $\beta-\mathrm{H}$ 消 除的 Heck 反应产物 19. 更有意思的是, 研究人员发现 在使用不同的氧化剂进行氧化时, 得到的最终产物也各 不相同. 例如采用醋酸铜和氧气氧化时, 得到的是 $\beta-\mathrm{H}$ 消除的 2,3-糖烯碳苷 19, 采用苯醌(BQ)作氧化剂时, 得 到的是 3-羰基碳苷 21, 而采用 2,3-二氯-5,6-二氰基苯醌 (DDQ)作氧化剂时, 得到的是烯酮类碳苷 20 (Scheme 2). 作者推测反应发生的主要原因是因为在使用 DDQ 或者 $\mathrm{BQ}$ 作氧化剂时，引起了 3-OH 上硅基保护基的水解. 作 者提出反应的立体选择性取决于 3 位取代基的立体构 型 ${ }^{[14]}$, 因为大位阻的活性 Pd 物种对糖烯双键进行加成 时，更倾向于从空间位阻更小的 3 位取代基的另一面进 行加成.

$$
\text { (1) }
$$

图式 $2 \mathrm{Pd}$ 催化的不同氧化剂存在下的糖烯与芳基硼酸的偶 联反应

Scheme 2 Reactions of glycals and arylboronic acids catalyzed by Pd with different oxidants

然而，当 Mukherjee 等 ${ }^{[15]}$ 在使用 TEMPO 作为氧化 剂时, 却能发生 Heck-Suzuki 类双偶联反应, 得到 cis1,2-二芳基化的碳苷产物 22 (Eq. 4). 研究人员分析反应 发生的主要原因可能是因为四甲基六氢吡啶氧化物 (TEMPO)本身所具有的自由基氧化特性, 能够使 Pd 配 位上去后连续发生两次氧化加成反应(Scheme 3).

除了芳基硼酸以外，芳基碘代物 ${ }^{[14]}$ 、芳基溴代物 ${ }^{[16]}$
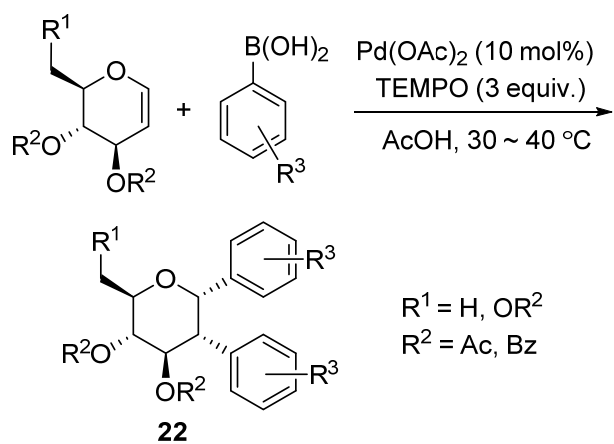

$\alpha$ only, 18 examples $65 \% \sim 80 \%$
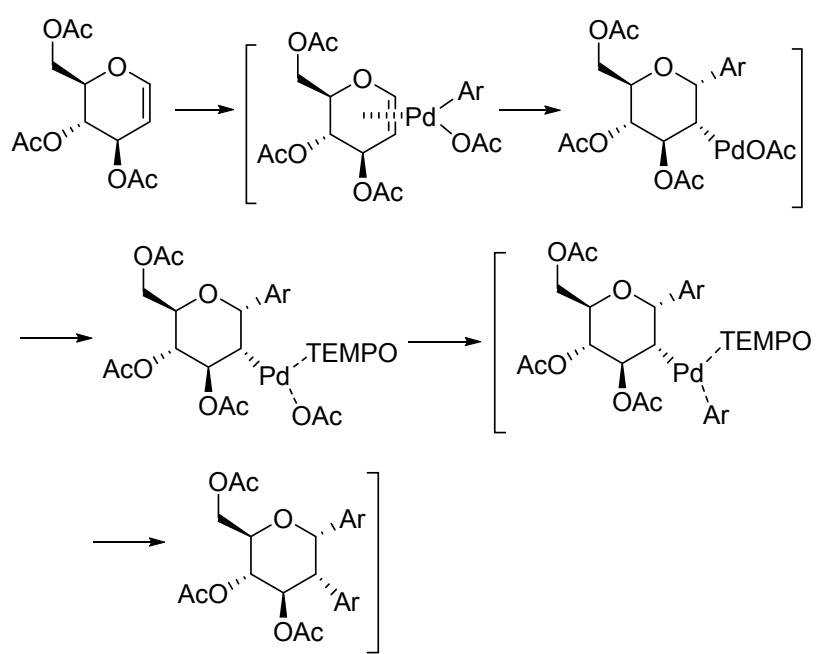

图式 $3 \mathrm{Pd}$ 催化的以 TEMPO 为氧化剂的糖烯与芳基嗍酸的 偶联反应机理

Scheme 3 Plausible mechanism for the reactions of glycals and arylboronic acids catalyzed by Pd with TEMPO as the oxidant

和烯醇磺酸酯 ${ }^{[17]}$ 同样也能在 Pd 催化下与糖烯发生 Heck 偶联反应(Scheme 4).

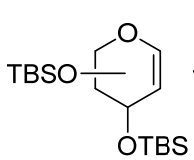
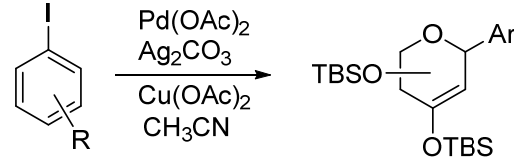

$\alpha$ only, 16 examples<smiles>BrC(Br)(Br)c1ccccc1</smiles>

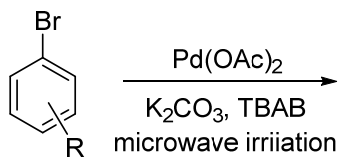
$59 \% \sim 99 \%$

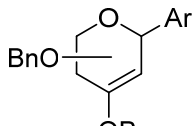
$\alpha$ only, 9 examples $73 \% \sim 81 \%$

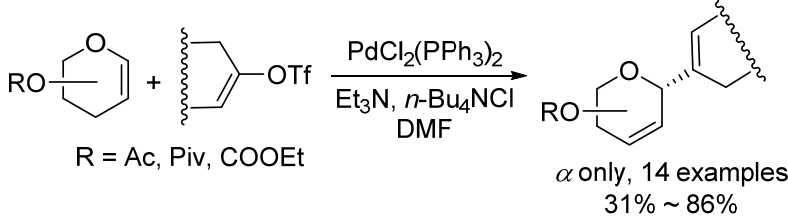

图式 4 Pd 催化的糖烯与其他芳基试剂的偶联反应 Scheme 4 Reactions of glycals and other aryl reagents catalyzed by Pd 
Dunkerton 等 ${ }^{[18]}$ 发现除了 1,2-不饱和糖烯化合物外, 2,3-不饱和糖烯化合物在 $\operatorname{Pd}(0)$ 的催化下能够形成 $\operatorname{Pd}-\pi$ 烯丙基的复合物, 而亲核试剂在该条件下能够直接与该 复合物发生亲核加成反应生成对应的 $C$-糖苷. 不久, Sinou 等 ${ }^{[19]}$ 还发现, 通过控制参加反应的配体的种类和 配比, 可以完全得到单一构型的 $C$-苷产物(Scheme 5).

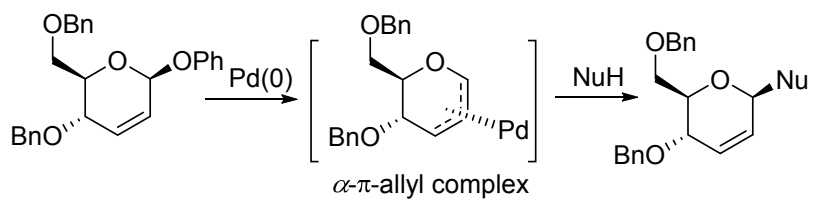

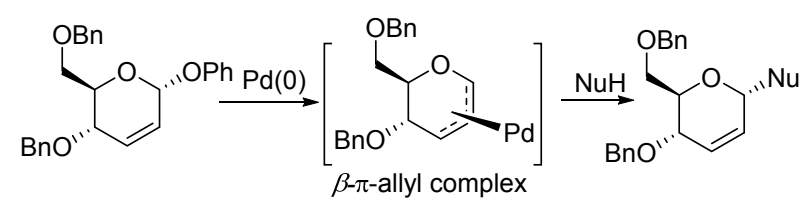

图式 $5 \mathrm{Pd}$ 催化的 2,3-不饱和糖烯与亲核试剂的偶联反应 Scheme 5 Reactions of 2,3-unsaturated glycals and nucleophiles catalyzed by Pd

\section{$1.2 \mathrm{Pd}$ 催化的 1-取代糖烯参与的偶联反应制备 $C$-苷}

早在 1990 年, Friesen 等 ${ }^{[20]}$ 就发现糖烯的 1-锡基取 代化合物与芳基澳化物能够在 Pd 催化下发生偶联反应, 生成对应的芳基糖烯碳苷. 芳基澳的对位或邻位有吸电 子取代基更有利于此反应的进行，同时反应的副产物主 要是糖烯的自身偶联产物(Eq. 5).

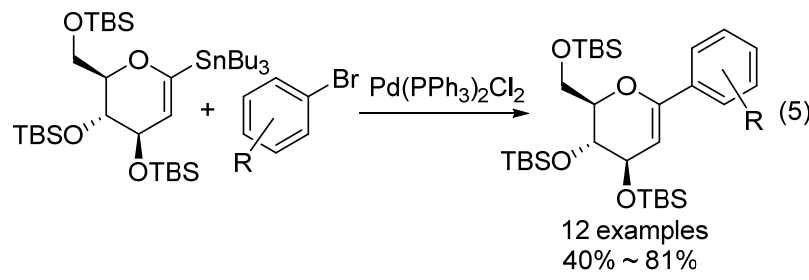

此外, 糖烯的金属衍生物, 如糖烯铟化合物 ${ }^{[21]}(\mathrm{Eq}$. 6)和糖烯锌化合物 ${ }^{[22]}$ (Scheme 6), 也能够与芳基卤化物 在 Pd 的催化下合成糖烯碳苷. Augé 等 ${ }^{[23]}$ 还将此方法用 于克级 $C$-糖肽的合成中(Eq. 7).

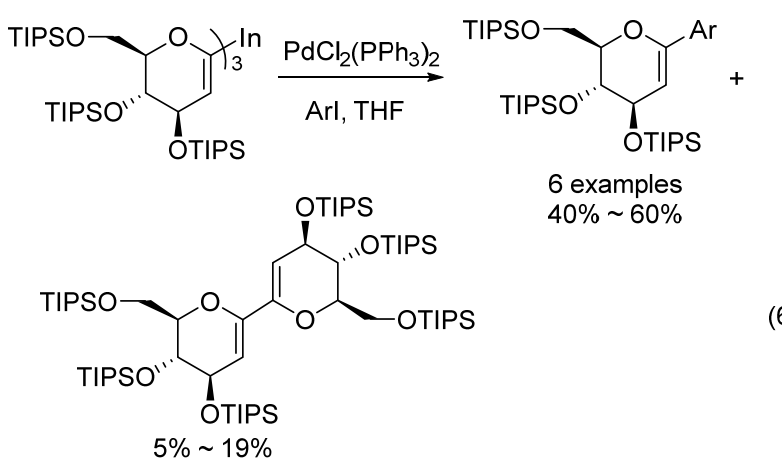

除了芳基卤化物外, 2010 年, Werz 等 ${ }^{[24]}$ 利用 1 -碘代 糖烯或 1-三氟甲磺酸酯糖烯(23)与糖基炔化物 24 在 Pd

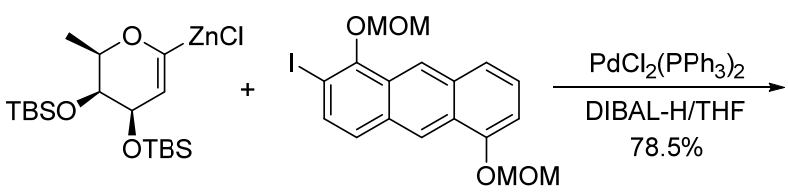

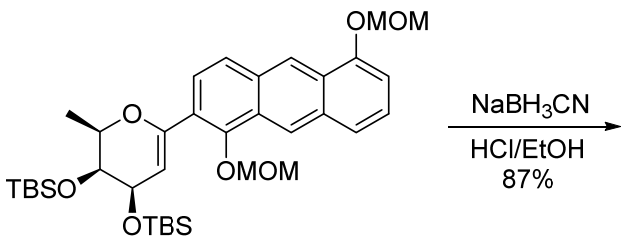

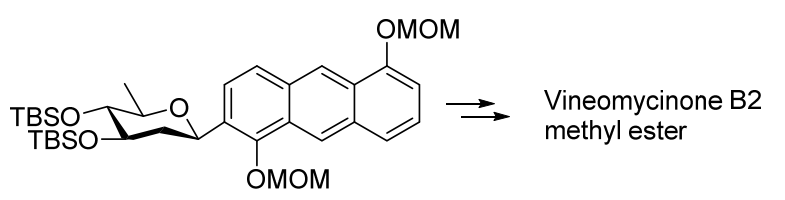

图式 $6 \mathrm{Pd}$ 催化的糖烯锌化合物与芳基碘试剂的偶联反应 Scheme 6 Reaction of zincated glycal and aryl iodide catalyzed by $\mathrm{Pd}$
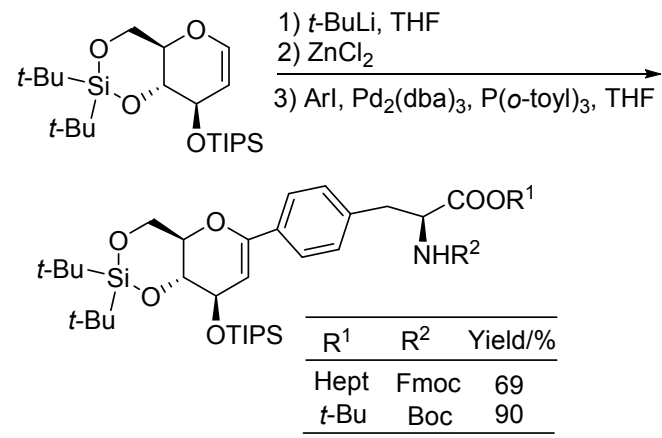

催化下, 合成了一系列 $1 \rightarrow 6$ 炔键连接的二糖碳苷 25 . 接着利用 Raney-Ni 可以选择性地将 $\mathbf{2 5}$ 中的连接键炔键 进行还原, 生成对应的糖烯碳苷 26. 并且在将糖烯的不 饱和双键进行氧化还原时, 除了可以使用传统的硼氢化 氧化得到 1,2 反式的 $\beta$ - $C$-苷 27 外 ${ }^{[25]}$, 研究人员还发现, 使用氧化剂过氧丙酮 (DMDO) 可以立体专一性地生成 $\alpha$ 面的 1,2-环氧糖 $(>20: 1)$, 接着使用与环氧的氧原子具 有配位效用的还原剂二异丁基氢化铝(DIBAL)则可以选 择性地得到 $\beta$ - $C$ - 苷 27 , 而使用位阻更小的超氢( $(\mathrm{LiBH}-$ $\mathrm{Et}_{3}$ ) 则可以得到具有相反构型的 $\alpha$ - $C$-苷 28 (Scheme 7).

随后, Werz 等 ${ }^{[26]}$ 又利用糖基锡试剂与环外的溴代烯 烃在 Pd 的催化下, 发生 Still 偶联得到连二烯型的碳苷 30, 通过这一反应可以快速地制备出各种 $(1 \rightarrow 2),(1 \rightarrow 3)$ 和 $(1 \rightarrow 4) \mathrm{C}-\mathrm{C}$ 键连接的二糖. 这种连二烯的结构可以 进一步还原生成 2-脱氧碳苷 31, 也可以通过嗍氢化氧 化制备 2-OH 碳苷 32 (Scheme 8).

\section{$1.3 \mathrm{Pd}$ 催化的 1-取代饱和糖基参与的偶联反应制备 $C$ - 苷}

除了 1-取代糖烯外，饱和的 1-锡基取代的糖基化合 物也能够与芳基卤化物发生偶联反应. 1995 年, Falck 
<smiles>[R]OCC1CC([R6])CCC1[Y]</smiles>

23

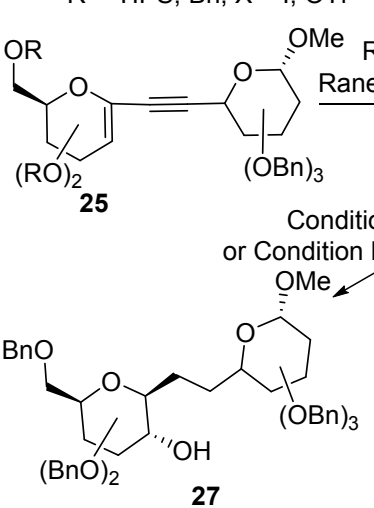

27
$\mathrm{R}=$ TIPS, Bn; $\mathrm{X}=\mathrm{I}$, OTf

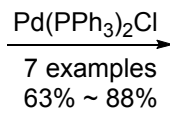$$
24
$$

$\mathrm{Me}$

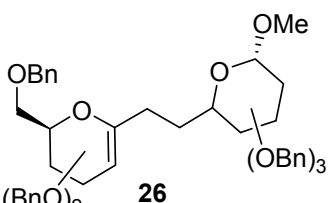
$\mathrm{OBn})_{3}$

Condition A: 1) DMDO, DCM; 2) DIBAL, DCM Condition B: 1) $\mathrm{BH}_{3} \cdot \mathrm{THF} ; 2$ ) $\mathrm{H}_{2} \mathrm{O}_{2}$, DCM Condition C: 1) DMDO, DCM; 2) $\mathrm{LiBHEt}_{3}$, THF

图式 $7 \mathrm{Pd}$ 催化的 $1 \rightarrow 6$ 连接碳苷二糖的制备 Scheme 7 Synthesis of $(1 \rightarrow 6)$-linked $C$-glycosides catalyzed by $\mathrm{Pd}$

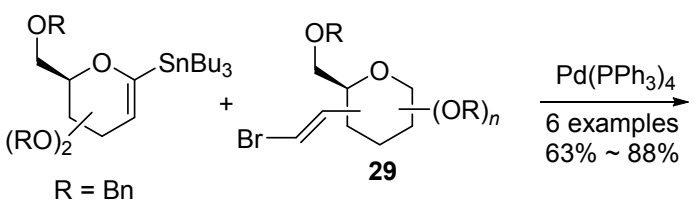

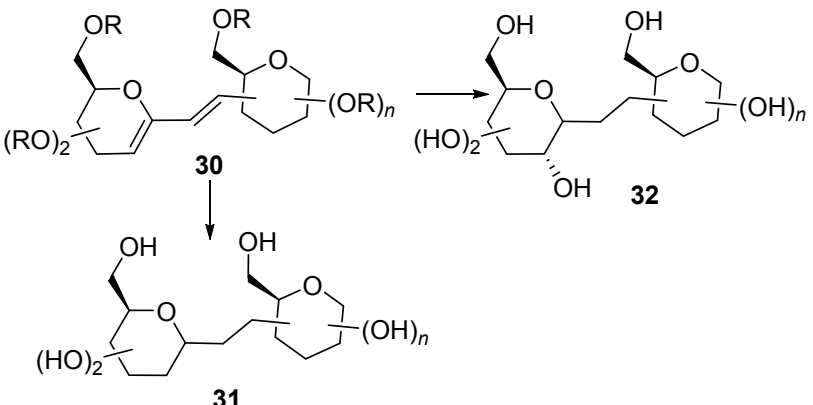

图式 $8 \mathrm{Pd}$ 催化的 $(1 \rightarrow 2),(1 \rightarrow 3)$ 和 $(1 \rightarrow 4)$ 连接的碳苷二糖的制 备

Scheme 8 Synthesis of $(1 \rightarrow 2),(1 \rightarrow 3)$ and $(1 \rightarrow 4)$-linked $C$-glycosides catalyzed by $\mathrm{Pd}$

等 ${ }^{[27]}$ 利用糖基 1 -锡基取代物 33 与酰氯在 $\mathrm{Pd} / \mathrm{Cu}$ 共催化 作用下, 制备出了 $\alpha$-羰基碳苷 34. 其中化合物 33 中糖 基 2 位的甲氧基甲基(MOM)保护基非常重要, 因为 MOM 保护基可以有效地稳定反应过程中生成的有机铜 中间体, 有利于异头位 $\mathrm{C}-\mathrm{C}$ 键的构建, 而改用简单的 醚键保护时, 例如 $\mathrm{Me}$ 或者 $\mathrm{Bn}$ 保护时, 则不会有此效果, 原料在这种条件下会发生降解(Eq. 8).

2016 年, Walczak 等 ${ }^{[28]}$ 也发现 1-锡基取代的糖基化 合物与芳基卤化物在 $\mathrm{Pd}$ 催化下能发生偶联反应, 生成
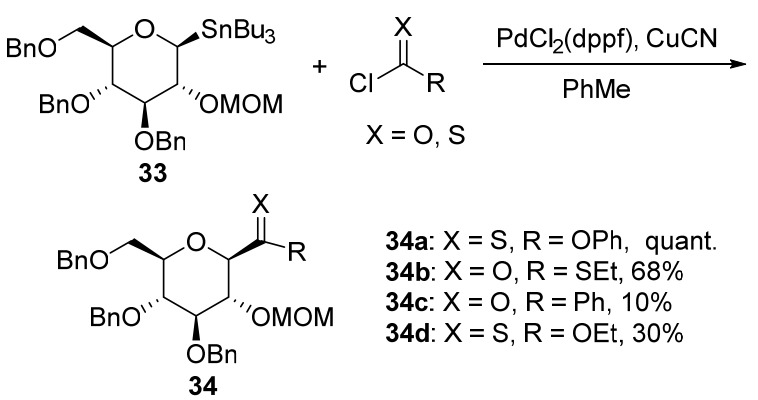

芳基碳苷. 这一反应最大的一个特色也就是可以通过控 制底物糖基锡的立体构型来控制生成的产物芳基碳苷 的立体构型，产物异头位的立体构型与原料糖基锡的立 体构型保持一致(Eq. 9).

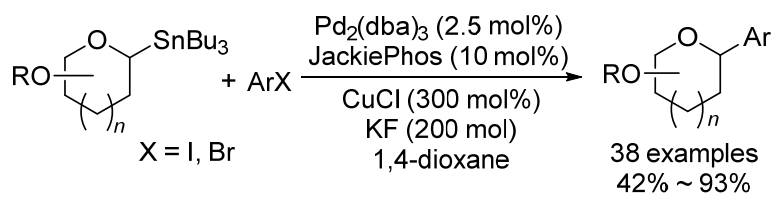

\section{$1.4 \mathrm{Pd}$ 催化的 1-取代糖烯参与的 $\mathrm{CO}$ 插入反应制备 C-苷}

1997 年, Vogel 等 ${ }^{[29]}$ 发现在 CO 存在下, 1-碘代或者 1-三丁基锡代糖烯能够与对应的烯烃锡化物或者卤化 物发生 CO 插入的偶联反应，生成对应的 $\alpha$-羰基糖烯碳 苷(Eq. 10).

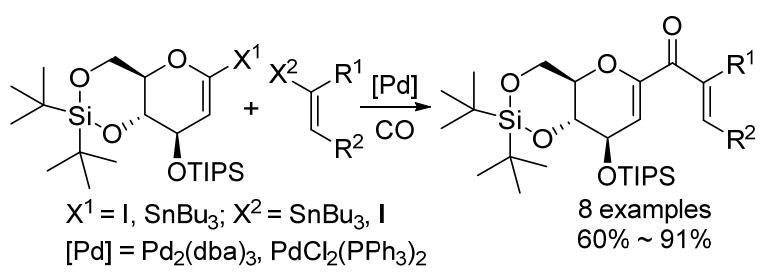

随后，Tan 研究小组 ${ }^{[30]}$ 在此基础上也发现 1-碘代糖 烯与原位生成的末端烯烃的硼酸酯也同样能够在 Pd 催 化下生成糖烯碳苷. 并且该反应在 $\mathrm{CO}$ 存在下，也能发 生 $\mathrm{CO}$ 插入反应，生成 $\alpha$-羰基糖烯碳苷(Scheme 9).

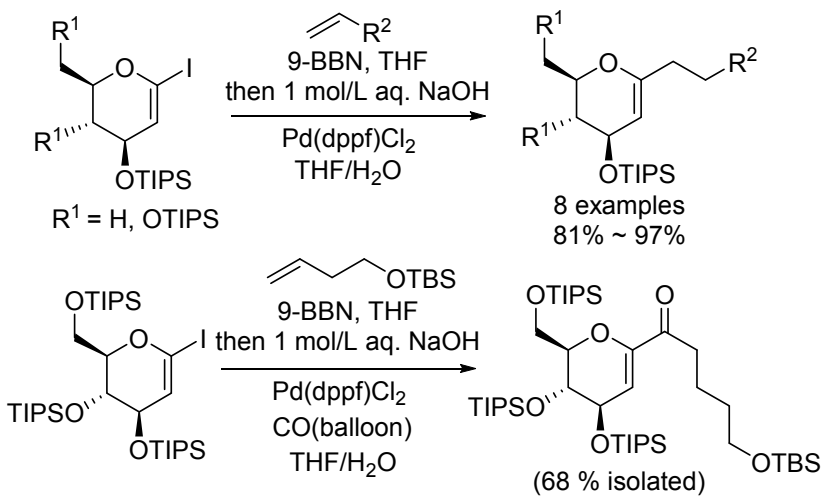

图式 $9 \mathrm{Pd}$ 催化的 C(1)-烷基或羰基碳苷的合成 Scheme 9 Synthesis of C(1)-alkyl- and acyl-glycals catalyzed by Pd 


\section{$1.5 P d$ 催化的 C-H 活化制备 C-苷}

过渡金属催化的导向基团诱导的 $\mathrm{C}-\mathrm{H}$ 键直接官能 团化在过去的几十年里已经成为构建 $\mathrm{C}-\mathrm{C}$ 键的重要方 法之一 ${ }^{[31]} .2016$ 年, $\mathrm{Ye}$ 等 ${ }^{[32]}$ 发展了 $\mathrm{Pd}$ 催化的芳基 $\mathrm{C}-\mathrm{H}$ 键活化直接与碘代糖烯进行偶联制备芳基碳苷的方法. 该方法采用 $N$-喹啉基作为反应的导向基团, 可以选择 性地活化苯甲酸类化合物的酰基邻位 $\mathrm{C}\left(\mathrm{sp}^{2}\right)-\mathrm{H}$ 键. 反 应的副产物主要是二糖苷化产物, 但是通过调节参与反 应的氨基酸配体, 可以主要得到单糖苷化产物(Eq. 11).
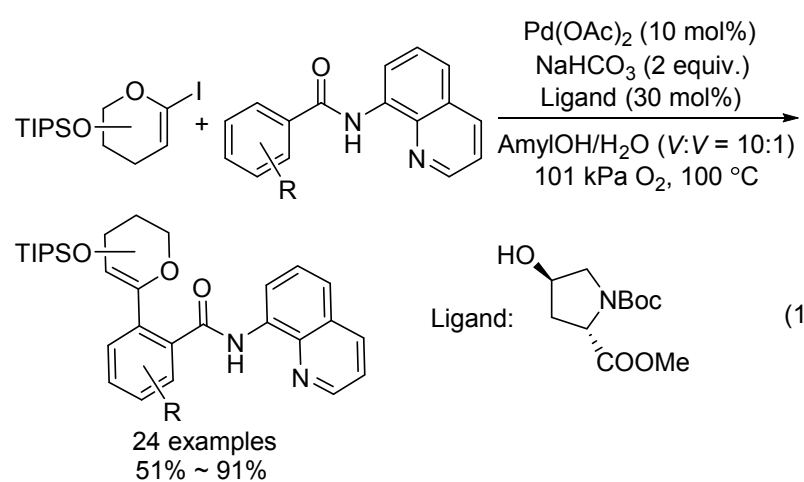

\section{$1.6 \mathrm{Pd}$ 催化的脱翔偶联反应制备 $C$-苷}

自 2002 年, Myers 等 ${ }^{[33]}$ 报道首例 Pd 催化的脱羒偶 联反应以来, 过渡金属催化的交叉脱羧偶联反应就开始 广泛应用于有机合成领域 ${ }^{[34]} .2011$ 年, Liu 等 ${ }^{[35]}$ 首次发现 芳基甲酸在 Pd 催化下也能够与糖烯发生脱羧偶联反应, 生成立体专一性的 $\alpha$-芳基碳苷. 与前述的 Heck 偶联反 应相比, 该类型反应在底物糖烯羟基上的保护基可选择 范围更广一些，除了酯基、苄基、硅基外还包括叔丁氧 羰基和苄叉等保护基(Eq. 12).

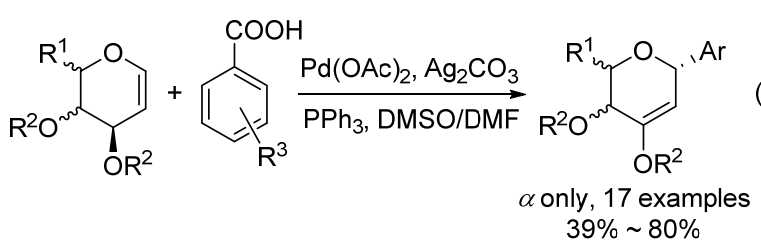

随后，该研究小组 ${ }^{[36]}$ 又发现糖环上的3-位酯基也能 够在 Pd 催化下, 发生分子内的脱羧重排反应生成 $C$-苷. 与分子间的脱羧偶联反应及传统的 Pd 催化的 Heck 偶联 产物不同的是，该类反应产物的立体构型主要为 $\beta$ 构型 (Eq. 13). 通过利用这一高立体选择性的 $C$-糖苷键构建 方法, 该小组 ${ }^{[37}$ 快速地完成了抗肿瘤活性小分子 Aspergillide $\mathrm{A}^{\mathrm{l}}$ 的合成.<smiles>[R]C(=O)O[C@H]1C=CO[C@H](CO)[C@H]1[R6]</smiles>

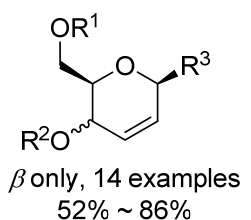

除了芳基羧酸可以与糖烯发生脱羧偶联外，芳基磺 酸钠 ${ }^{[38]}$ 和芳基磺酰氯 ${ }^{[39]}$ 同样也能够在 Pd 催化下与糖烯 发生脱磺酸偶联反应生成芳基碳苷(Scheme 10).
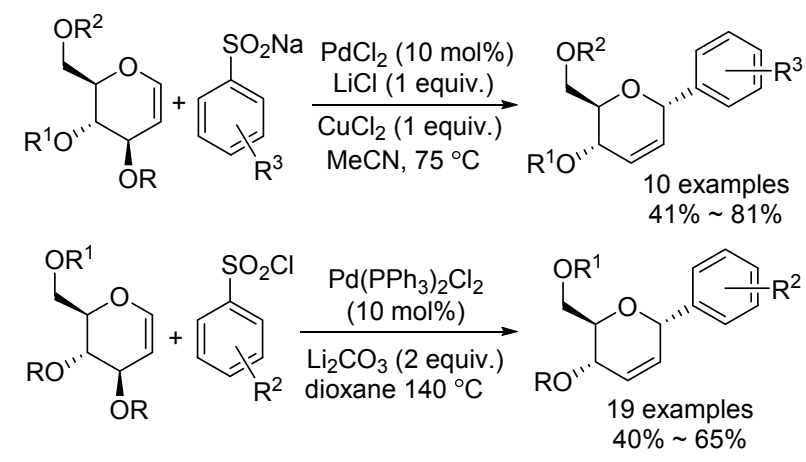

图式 $10 \mathrm{Pd}$ 催化的利用芳基磺酸化合物制备碳苷 Scheme 10 Synthesis of $C$-glycosides from glycals and arylsulfonyl sodium (or chloride)

除脱羧外，芳基肼与糖烯在 Pd 催化下也能够发生 $\mathrm{C}-\mathrm{N}$ 键的断裂, 生成芳基 $C$-苷 ${ }^{[40]}$. 但是研究人员发现, 当糖烯 3 位为 $R$ 构型时, 能够得到立体转移性的 $\alpha$-构型 产物; 而 3 位为 $S$ 构型时, 得到的却是 $\alpha, \beta$ 的混合物 (Scheme 11). 作者由此认为反应的立体选择性并不如 $\mathrm{Ye}$ 等 ${ }^{[14]}$ 预测的一样仅仅取决于 3 位取代基的立体构型.
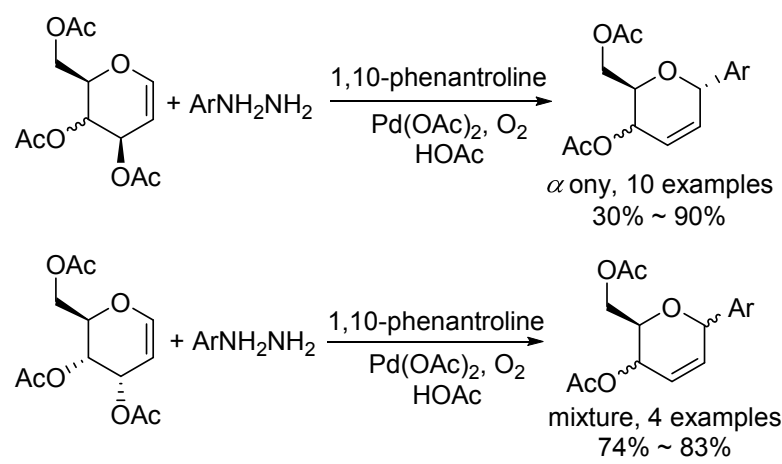

图式 $11 \mathrm{Pd}$ 催化的糖烯与芳基肼反应

Scheme 11 Reactions of glycals and aryl hydrazines catalyzed by Pd

\section{$1.7 \mathrm{Pd}$ 参与的共催化反应制备 $C$-苷}

自从 Trost 等 ${ }^{[41]}$ 利用钯与嗍共催化实现了醇对环氧 化合物的高效开环反应以来, 共催化在有机合成中也得 到了非常广泛的应用，它实现了传统催化反应不能实现 的新的化学转化和高效不对称催化等. 例如 Nicolaou 等 ${ }^{[42]}$ 利用 $\mathrm{Pd} / \mathrm{Cu}$ 共催化 1-锡基取代的糖烯化合物与烯醇 磺酸酯发生偶联, 生成 $C$-苷(Eq. 14).

2014 年, Liu 等 ${ }^{[43]}$ 也利用共催化的方法实现了糖烯 和 2-吡啶甲醛的偶联反应，生成 $\alpha$-羰基碳苷。他们采用 过渡金属 $\mathrm{Pd}$ 和有机小分子 3,4-二甲基-5-(2-羟乙基)碘代 


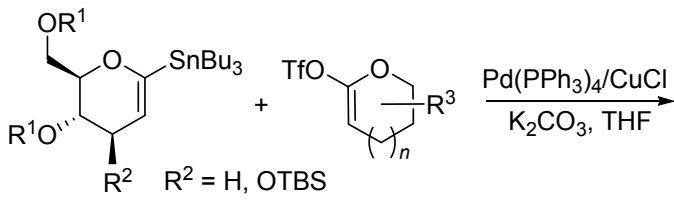

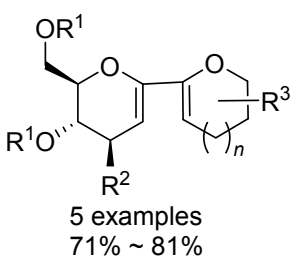

噻唑(NHC))作为共催化剂, 其中, Pd 与糖烯形成亲电性 的 Pd- $\pi$-烯丙基复合物, 而 $\mathrm{NHC}$ 活化 2-醛基吡啶形成亲 核性的 Breslow 中间体，随后两种中间体发生加成反应 生成糖烯碳苷(Scheme 12).<smiles>[R]C1OC[C@H]2OC=C[C@H](OOCC)[C@H]2O1</smiles><smiles></smiles>

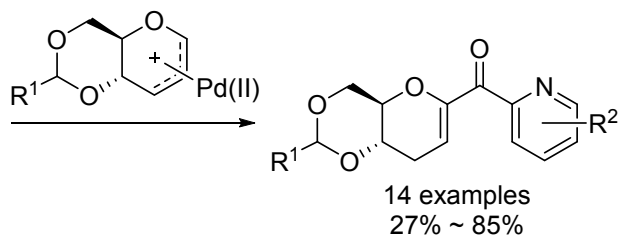

图式 $12 \mathrm{Pd} / \mathrm{NHC}$ 共催化的糖烯与芳基醛反应 Scheme 12 Reactions of glycals and (o-azaaryl) carboxaldehyde co-catalyzed by Pd and NHC

\section{$2 \mathrm{Ni}$ 催化的偶联反应制备 $\mathrm{C}$-苷}

虽然金属 Pd 催化的偶联反应在糖烯类型的 $C$-苷合 成中较为多见，但是对于 $\mathrm{C}(1)$-卤素取代的糖苷来说，由 于反应中生成的 $\mathrm{C}(1)-\mathrm{Pd}$ 键非常容易发生 $\beta-\mathrm{H}$ 消除, 因 此很难通过 Pd 催化的偶联反应来构建 1,2 饱和的 $C$-糖 苷键. 2000 年, Marsden 等 ${ }^{[44]}$ 发现糖基溴苷与缺电子的 末端烯烃在 $\mathrm{Ni}$ 催化下, 可以发生偶联反应生成 $C$-苷. 作者推测反应发生的可能历程是糖基溴苷在过渡金属 $\mathrm{Ni}(\mathrm{II})$ 和 $\mathrm{Mn}$ 共催化作用下形成了糖基自由基 ${ }^{[45]}$ ，随后该 糖基自由基与双键进行加成后得到 $\alpha$ 构型占主要的烷基 $C$-苷. 其中当 $\mathrm{R}^{1}$ 为酯基取代时, 反应具有非常好的 $\alpha$ 构 型立体选择性 $(36 \mathrm{a}, 36 \mathrm{~b})$, 但是 $\mathrm{R}^{1}$ 为氰基取代时, 却还 得到了少量 $\beta$ 构型的产物(36c). 而当 $\mathrm{R}^{1}$ 为苯基取代时, 没有任何目标 $C$-苷的生成(36d) (Eq. 15).

2007 年, Gagne 等 ${ }^{[46]}$ 又发现糖基溴苷或者氯苷与烷

$$
\begin{array}{cccccc}
\text { 36 } & \mathrm{X} & \mathrm{Y} & \mathrm{R}^{1} & \mathrm{R}^{2} & \mathrm{Yield} / \% \\
\hline 36 \mathrm{a} & \mathrm{H} & \mathrm{OAc} & \mathrm{CO}_{2} \mathrm{Me} & \mathrm{Me} & \alpha, 98 \% \\
\mathbf{3 6} & \mathrm{OAc} & \mathrm{H} & \mathrm{CO}_{2} \mathrm{Me} & \mathrm{Me} & \alpha, 98 \% \\
\mathbf{3 6 c} & \mathrm{H} & \mathrm{OAc} & \mathrm{CN} & \mathrm{H} & \alpha, 76 \% ; \beta, 5 \% \\
\mathbf{3 6 d} & \mathrm{H} & \mathrm{OAc} & \mathrm{Ph} & \mathrm{H} & \mathrm{NR} \\
\hline
\end{array}
$$

基锌试剂在 $\mathrm{NiCl}_{2}$ 的催化下，室温下就能发生 Negishi 偶 联，生成烷基碳苷产物. 该类型反应具有较好的底物普 适性，且反应产物在甘露糖底物上具有非常好的 $\alpha$ 型立 体选择性，但是遗憾的是对于葡萄糖型的底物，立体选 择性并不是太好，反应的副产物主要是糖烯(Eq. 16).

$$
\text { ( }
$$

紧接着，在进一步的研究过程中他们发现当使用 $\mathrm{Ni}(\mathrm{COD})_{2}$ 作为催化剂时，通过篮选合适的配体 $t$-Bu-Terp，除了烷基锌试剂外，芳基锌试剂同样也能发 生偶联反应，并且该类型反应在葡萄糖型底物上具有非 常好的 $\beta$ 型立体选择性 ${ }^{[47]}$ (Eq. 17).

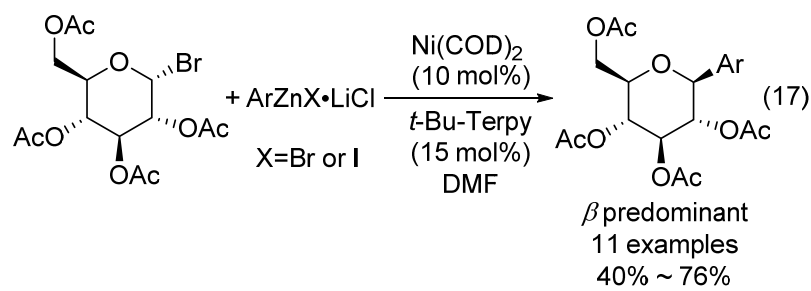

除了烷基和芳基锌试剂外，缺电子烯烃也能够与葡 萄糖溴苷在锌粉的还原下，通过 $\mathrm{Ni}(\mathrm{COD})_{2}$ 催化发生偶 联反应得到 $\alpha$ 型 $C$-苷产物 ${ }^{[48]}(\mathrm{Eq} .18)$.

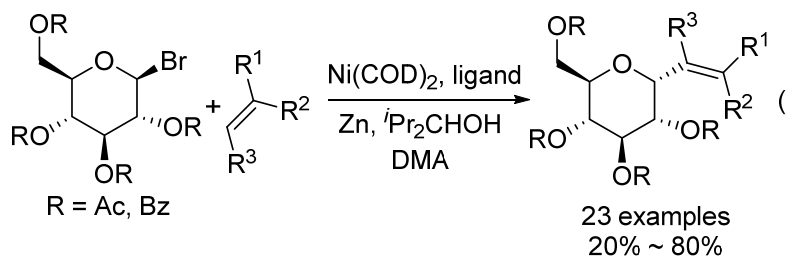


2014 年, Gong 等 ${ }^{[49]}$ 又利用糖基溴苷和酸通过 $\mathrm{Ni}$ 催 化下的还原偶联反应得到了 $\alpha$-羰基碳苷. 在产物的立体 选择性方面, 对于甘露糖底物得到了完全 $\alpha$ 构型的产物, 而对于葡萄糖和半乳糖型的底物, 也是以 $\alpha$ 构型为主 (Eq. 19).

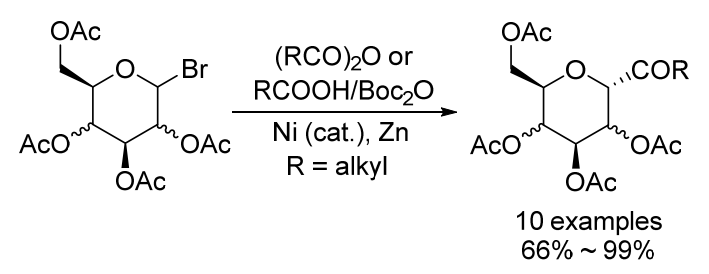

除了 $\alpha$-羰基碳苷外, 他们还利用糖基溴苷与氯甲酸 酯在 $\mathrm{Ni}$ 的催化下进行还原偶联反应, 制备得到了 $\alpha$-酯 基碳苷 ${ }^{[50]}$ (Eq. 20).

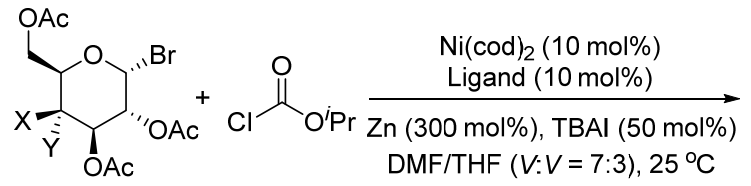

$$
\begin{aligned}
& \text { Ligand: } \\
& \mathrm{X}=\mathrm{H}, \mathrm{Y}=\mathrm{OAc}, 70 \%, \alpha \cdot \beta=2.6: 1 \\
& \mathrm{X}=\mathrm{H}, \mathrm{Y}=\mathrm{OAc}, 65 \%, \alpha . \beta=5.8: 1
\end{aligned}
$$

而 $\mathrm{Ye}$ 等 ${ }^{[51]}$ 在尝试使用糖烯与芳基碀酸酯或芳基三 氟硣酸钾在 $\mathrm{Ni}$ 催化下进行偶联反应时, 只得到了开链 的 $C$-苷产物 37. 但是他们在进一步的研究过程中发现, 该产物在酸等关环反应的条件下，能够分子内关环生成 所需要的碳苷产物, 并且当采用不同的关环条件时, 可 以得到结构完全不同的关环碳苷产物. 例如在使用路易 斯酸 $\mathrm{Sc}(\mathrm{OTf})_{3}$ 关环时, 主要得到 $\beta$ 构型的 2,3-不饱和 $C$ 苷, 而采用质子酸 $\mathrm{PhPHBr}$ 关环时, 却主要得到 $\alpha$ 构型 的 2,3-不饱和 $C$-苷. 在采用 $\mathrm{PhSeCl} / \mathrm{Bu}_{3} \mathrm{SnH}$ 时得到 2-脱 氧的 $\beta$ - $C$-苷 $(40 \mathbf{a}, 40 \mathrm{~b})$, 而采用 $\mathrm{NBS}$ 或 NIS $/ \mathrm{Bu}_{3} \mathrm{SnH}$ 时 得到的却是 2-脱氧的 $\alpha$ - $C$-苷 42 (Scheme 13).

\section{3 其他金属催化的偶联反应制备 C-苷}

2012 年, Cossy 等 ${ }^{[52]}$ 发现在金属 Co 催化下, 芳基格 氏试剂或烯基格氏试剂能够与糖基溴苷发生亲核取代 反应, 生成芳基碳苷(Eq. 21). 由于 Co 不易发生 $\beta$ - H 消 除，因此反应几乎不会生成糖烯的副产物. 同时该类型 反应在全乙酰化的 $\alpha$-溴代甘露糖和半乳糖底物上具有 非常好的 $\alpha$ 选择性, 但是在葡萄糖底物上的立体选择性 较为一般.

2007 年, Somsák 等 ${ }^{[53]}$ 发现利用糖基溴苷与缺电子

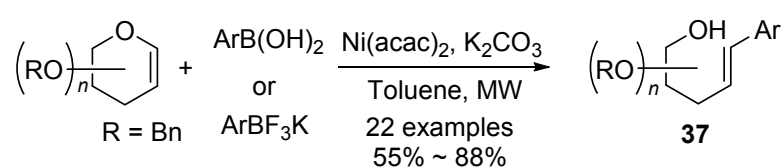
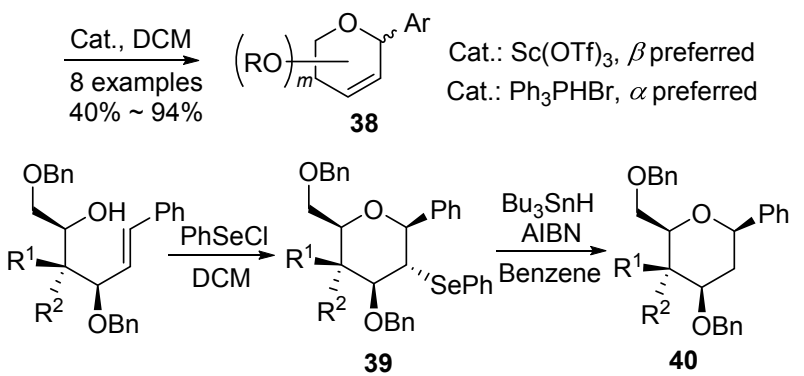

39a $\mathrm{R}^{1}=\mathrm{H}, \mathrm{R}^{2}=\mathrm{OBn}, 85 \%$

40a $R^{1}=H, R^{2}=O B n, 93 \%$ 39b $R^{1}=O B n, R^{2}=H, 88 \%$

40b $R^{1}=O B n, R^{2}=H, 87 \%$

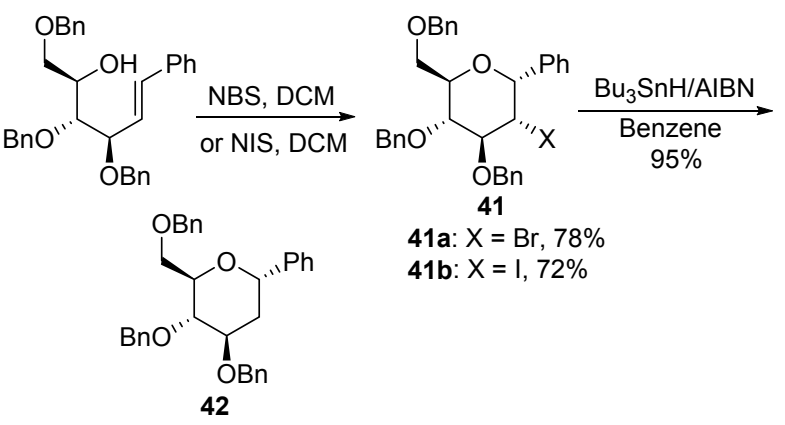

图式 $13 \mathrm{Ni}$ 催化的糖烯与芳基硼酸反应制备 $C$-苷

Scheme 13 Synthesis of $C$-glycosides from glycals and aryl boronic acid catalyzed by $\mathrm{Ni}$

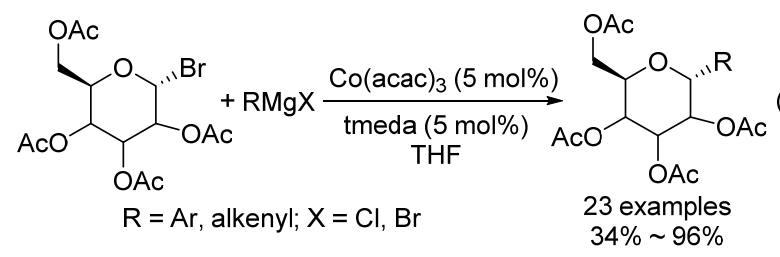

的末端烯烃在 $\mathrm{Cr}$ 的催化下, 能够发生加成反应生成 $C-$ 苷. 但是只有在底物为葡萄糖时, 产物不仅具有较好的 $\alpha$-选择性, 产率也较高 $(46 \mathrm{a} \sim 46 \mathrm{c})$. 当底物为半乳糖和 木糖时, 虽然反应仍然具有很好的立体选择性, 但是产 率都不是太高 $(47 \mathrm{a}, 48 \mathrm{~b})$; 但是当底物为阿拉伯糖时, 得 到的却是 $\alpha, \beta$ 的混合物 $(\mathbf{5 0 a}, \mathbf{5 0 b}, \alpha: \beta=1: 1$ ) (Scheme 14).

2010 年, Gagné 等 ${ }^{[54]}$ 发现糖基溴苷能够与末端烯烃 在金属 $\mathrm{Ru}$ 催化下, 发生偶联反应生成 $\alpha$ - $C$-苷. 研究表 明反应中 Hantzsch ester 的使用对反应产率的提高非常 重要, 可以在很大程度上抑制糖二聚产物的生成(Eq. 22).

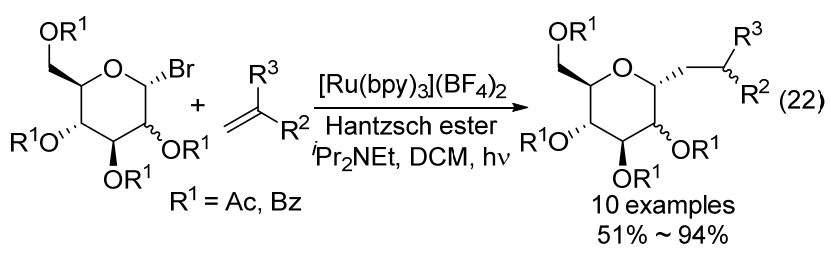




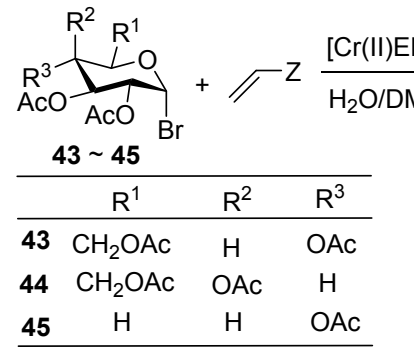

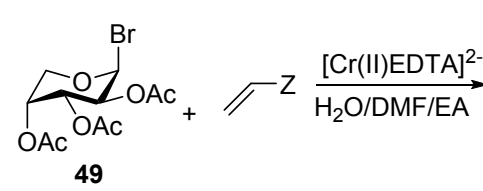

46a: $81 \%, Z=C N$

46b: $76 \%, Z=\mathrm{COOMe}$

46c: $52 \%, Z=\mathrm{COOH}$

47a: $29 \%, Z=C N$

48b: $21 \%, Z=$ COOMe

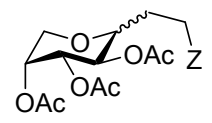

50a: $84 \%, Z=C N$

50b: $53 \%, Z=$ COOMe

图式 $14 \mathrm{Cr}$ 催化的糖基溴苷与烯烃反应制备 $C$-苷 Scheme 14 Synthesis of $C$-glycosides from glycosyl bromides and alkenes catalyzed by $\mathrm{Cr}$

研究人员推测该反应是通过可见光诱导下的自由 基反应历程进行的，同时反应具有很好的立体选择性. 后续研究表明 $\mathrm{Ru}(\mathrm{dmb})_{3}{ }^{2+}$ 比 $\mathrm{Ru}(\mathrm{bpy})_{3}{ }^{2+}$ 具有更好的催化 活性 ${ }^{[55]}$, 作者还利用这一方法完成了 $C$-糖肽等的合 成 ${ }^{[56]}$ (Scheme 15).<smiles>[R]OC[C@H]1OC[C@@H]([R17])[C@@H](O)[C@H]1[R10][H]</smiles>

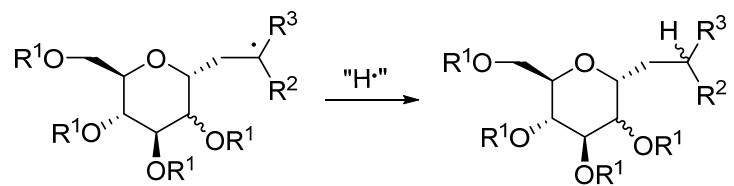<smiles>CCN(CC)C(C)SC(C)N(CC)C(C)C</smiles><smiles>CCOC(=O)C1=C(C)NC(C)=C(C(=O)OCC)C1</smiles>

图式 $15 \mathrm{Ru}$ 催化的糖基溴苷与烯烃反应制备 $C$-苷的机理 Scheme 15 A plausible mechanism for the synthesis of $C$-glycosides from glycosyl bromides and alkenes catalyzed by $\mathrm{Ru}$

2001 年, Maddaford 等 ${ }^{[57]}$ 利用金属 $\mathrm{Rh}$ 催化的芳基硼 酸和糖烯酮反应得到了立体专一性 $\alpha$ - $C$-苷, 且当芳基上 有给电子取代基时会更有利于反应的发生(Eq. 23).

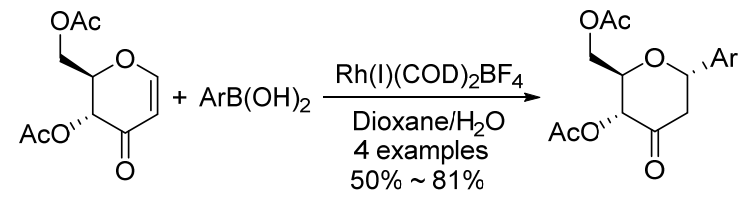

反应发生的可能机理如 Scheme 16 所示: 芳基硼酸 先与 $\mathrm{Rh}$ 催化剂发生转金属化, 接着芳基金属化合物对
糖烯酮发生 $\alpha$-面的加成反应，接着加成产物在水的存在 下进行水解即得到了 $\alpha-C$-苷. 研究表明反应中水的存在 对 $\mathrm{Rh}-\mathrm{O}$ 键的解离至关重要, 若在无水条件下进行反 应，几乎得不到目标产物 $(<5 \%)$.

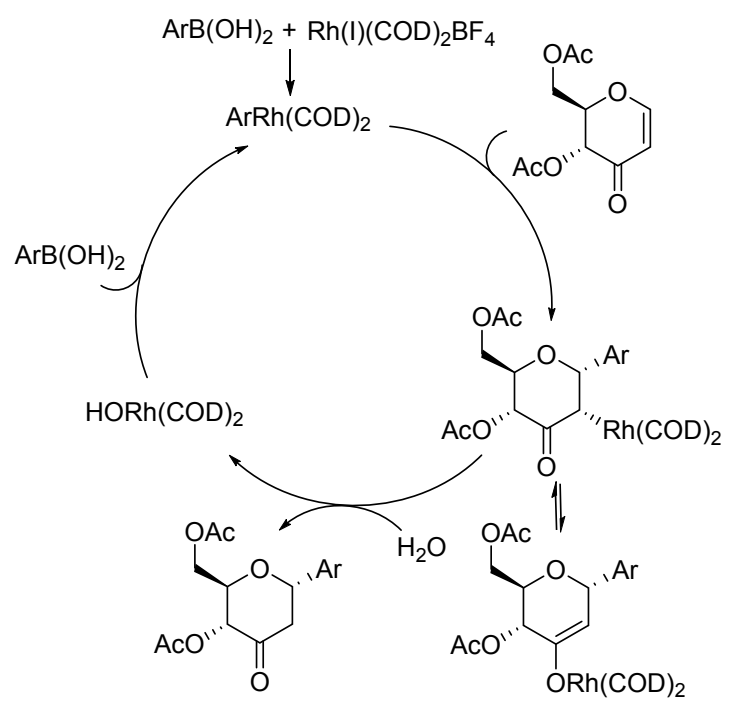

图式 $16 \mathrm{Rh}$ 催化的糖烯与芳基硼酸反应制备 $C$-苷的机理 Scheme 16 A plausible mechanism for the synthesis of $C$-glycosides from glycals and arylboronic acids catalyzed by $\mathrm{Rh}$

\section{4 总结与展望}

与传统的糖苷化反应相比，过渡金属催化的偶联反 应制备 $C$-苷具有立体选择性好, 产率高且反应多样化 的特点，同时它与传统糖苷化完全不同的催化体系也大 大丰富了寊糖合成中糖苗化正交策略的选择范围. 其中 在各类不同类型的异头位 $C$-苷的合成方面, 芳香 $C$-苷 和烯基 $C$-苷的研究相对来说比较多，而 $\alpha$-羰基 $C$-苷和 烷基 $C$-苷的合成研究还不是很多. 在反应的立体选择 性方面，目标产物立体构型的影响因素主要包括哪些， 以及如何更好地控制目标产物的立体构型还需要更进 一步的研究结果来证实. 在各类不同金属的催化活性方 面，金属 Pd 催化的偶联反应在制备 $C$-苷反应中已经得 到了较为广泛的应用，接着是 $\mathrm{Ni}$ 催化，但是其他过渡金 属催化的偶联反应制备 $C$-苷还是不多见. 总体来说，过 渡金属催化的偶联反应制备 $C$-糖苷的合成研究与现有 的偶联反应生成 $\mathrm{C}-\mathrm{C}$ 键的反应相比，还大有发展空间. 如何更加充分地利用过渡金属催化的偶联反应来制备 $C$-糖苷是糖化学工作者们接下来需要重点关注的一个 研究方向。

\section{Referenes}

[1] Zou, W. Curr. Top. Med. Chem. 2005, 5, 1363.

[2] Hultin, P. G. Curr. Top. Med. Chem. 2005, 5, 1299.

[3] (a) Cao, X.; Tian, Y.; Zhang, T.; Li, X.; Ito, Y. J. Chromatogr. A 1999, 855, 709. 
(b) Nomura, S.; Sakamaki, S.; Hongu, M.; Kawanishi, E.; Koga, Y.; Sakamoto, T.; Yamamoto, Y.; Ueta, K.; Kimata, H.; Nakayama, K.; Tsuda-Tsukimoto, M. J. Med. Chem. 2010, 53, 6355.

[4] Moore, R. E.; Scheuer, P. J. Science 1971, 172, 495.

[5] (a) De Clercq, E. J. Med. Chem. 2016, 59, 2301.

(b) Lalitha, K.; Muthusamy, K.; Prasad, Y. S.; Vemula, P. K.; Nagarajan, S. Carbohydr. Res. 2015, 402, 158.

(c) Yuan, X. J.; Linhardt, R. J. Curr. Top. Med. Chem. 2005, 5, 1393.

(d) Du, Y.; Linhardt, R. J.; Vlahov, I. R. Tetrahedron 1998, 54, 9913.

(e) Lee, D. Y. W.; He, M. S. Curr. Top. Med. Chem. 2005, 5, 1333.

(f) Wellington, K. W.; Benner, S. A. Nucleosides Nucleotides Nucleic Acids 2006, 25, 1309.

(g) Li, X.; Zhu, J. Eur. J. Org. Chem. 2016, 2016, 4724.

[6] (a) Yang, D.-C.; Zhang, Q.-H.; Xiong, W.-W.; Yuan, L.-G.; Cai, Q.-S.; Yang, M.-M.; Li, X.; Jiang, Y.-J.; Liu, Y.; Li, P.; Xu, Z.-S.; Sun, P.-P.; Geng, H.-L. Chin. J. Org. Chem. 2015, 35, 961 (in Chinese).

(袁定重, 张庆华, 廖世军, 熊文文, 元利刚, 蔡奇胜, 杨梦梅, 李雄, 蒋烨佳, 刘妍, 李萍, 徐贞帅, 孙盼盼, 耿会玲, 有机化 学, 2015, 35, 961.)

(b) Zhang, J.; Lu, Q.-Q.; Liu, C.; Lei, A.-W. Chin. J. Org. Chem. 2015, 35, 743 (in Chinese).

(张剑, 陆庆全, 刘超, 雷爱文, 有机化学, 2015, 35, 743.)

(c) Liu, J.; Zhu, Q.-R.; Du, J.-Z.; Xiu, L. Chin. J. Org. Chem. 2015, 35, 15 (in Chinese).

(刘杰; 朱庆仁; 杜娟张; 袖丽, 有机化学, 2015, 35, 15.)

(d) Zhang, W.-M.; Dai, J.-J.; Xu, H.-J. Chin. J. Org. Chem. 2015, 35, 1820 (in Chinese).

(张文曼, 戴建军, 许华建, 有机化学, 2015, 35, 1820.)

[7] Bergstrom, D. E.; Ruth, J. L. J. Am. Chem. Soc. 1976, 98, 1587.

[8] Arai, I.; Daves, G. D. J. Am. Chem. Soc. 1978, 100, 287.

[9] Daves, G. D. Acc. Chem. Res. 1990, 23, 201.

[10] Cheng, J. C. Y.; Daves, G. D. J. Org. Chem. 1987, 52, 3083.

[11] Ramnauth, J.; Poulin, O.; Rakhit, S.; Maddaford, S. P. Org. Lett. 2001, 3, 2013 .

[12] Beletskaya, I. P.; Cheprakov, A. V. Chem. Rev. 2000, 100, 3009.

[13] Xiong, D. C.; Zhang, L. H.; Ye, X. S. Org. Lett. 2009, 11, 1709.

[14] Li, H. H.; Ye, X. S. Org. Biomol. Chem. 2009, 7, 3855.

[15] Kusunuru, A. K.; Jaladanki, C. K.; Tatina, M. B.; Bharatam, P. V.; Mukherjee, D. Org. Lett. 2015, 17, 3742.

[16] Lei, M.; Gao, L.; Yang, J.-S. Tetrahedron Lett. 2009, 50, 5135.

[17] Bai, Y.; Leow, M.; Zeng, J.; Liu, X. W. Org. Lett. 2011, 13, 5648.

[18] Dunkerton, L. V.; Euske, J. M.; Serino, A. J. Carbohydr. Res. 1987, 171,89 .

[19] Brakta, M.; Lhoste, P.; Sinou, D. J. Org. Chem. 1989, 54, 1890.

[20] Friesen, R. W.; Sturino, C. F. J. Org. Chem. 1990, 55, 2572.

[21] Lehmann, U.; Awasthi, S.; Minehan, T. Org. Lett. 2003, 5, 2405.

[22] Tius, M. A.; Gomez-Galeno, J.; Gu, X. Q.; Zaidi, J. H. J. Am. Chem. Soc. 1991, 113, 5775.

[23] Ousmer, M.; Boucard, V.; Lubin-Germain, N.; Uziel, J.; Augé, J. Eur. J. Org. Chem. 2006, 1216.

[24] Koester, D. C.; Leibeling, M.; Neufeld, R.; Werz, D. B. Org. Lett. 2010, 12, 3934

[25] Schmidt, R. R.; Preuss, R.; Betz, R. Tetrahedron Lett. 1987, 28, 6591.

[26] Koester, D. C.; Kriemen, E.; Werz, D. B. Angew. Chem., Int. Ed. 2013, 52, 2985.
[27] Belosludtsev, Y. Y.; Bhatt, R. K.; Falck, J. R. Tetrahedron Lett. 1995, 36, 5881.

[28] Zhu, F.; Rourke, M. J.; Yang, T. Y.; Rodriguez, J.; Walczak, M. A. J. Am. Chem. Soc. 2016, 138, 12049.

[29] Jeanneret, V.; Meerpoel, L.; Vogel, P. Tetrahedron Lett. 1997, 38, 543.

[30] Potuzak, J. S.; Tan, D. S. Tetrahedron Lett. 2004, 45, 1797.

[31] (a) Lyons, T. W.; Sanford, M. S. Chem. Rev. 2010, 110, 1147. (b) Liu, C.; Zhang, H.; Shi, W.; Lei, A. Chem. Rev. 2011, 111, 1780.

[32] Liu, M.; Niu, Y.; Wu, Y. F.; Ye, X. S. Org. Lett. 2016, 18, 1836.

[33] Myers, A. G.; Tanaka, D.; Mannion, M. R. J. Am. Chem. Soc. 2002 124,11250 .

[34] Rodriguez, N.; Goossen, L. J. Chem. Soc. Rev. 2011, 40, 5030.

[35] Xiang, S.; Cai, S.; Zeng, J.; Liu, X. W. Org. Lett. 2011, 13, 4608.

[36] Zeng, J.; Ma, J.; Xiang, S.; Cai, S.; Liu, X. W. Angew. Chem., Int. Ed. 2013, 52, 5134.

[37] Kito, K.; Ookura, R.; Yoshida, S.; Namikoshi, M.; Ooi, T.; Kusumi, T. Org. Lett. 2008, 10, 225.

[38] Ma, J.; Xiang, S.; Jiang, H.; Liu, X.-W. Eur. J. Org. Chem. 2015, 2015, 949.

[39] Kusunuru, A. K.; Yousuf, S. K.; Tatina, M.; Mukherjee, D. Eur. J. Org. Chem. 2015, 2015, 459.

[40] Bai, Y.; Kim, L. M. H.; Liao, H.; Liu, X.-W. J. Org. Chem. 2013, $78,8821$.

[41] Trost, B. M.; McEachern, E. J.; Toste, F. D. J. Am. Chem. Soc. 1998, 120, 12702

[42] Nicolaou, K. C.; Sato, M.; Miller, N. D.; Gunzner, J. L.; Renaud, J.; Untersteller, E. Angew. Chem., Int. Ed. 1996, 35, 889.

[43] Bai, Y.; Leng, W. L.; Li, Y.; Liu, X. W. Chem. Commun. (Camb.) 2014, 50, 13391.

[44] Readman, S. K.; Marsden, S. P.; Hodgson, A. Synlett 2000, 1628.

[45] (a) Adlington, R. M.; Baldwin, J. E.; Basak, A.; Kozyrod, R. P. J. Chem. Soc., Chem. Commun. 1983, 944.

(b) Dunach, E.; Esteves, A. P.; Freitas, A. M.; Medeiros, M. J.; Olivero, S. Tetrahedron Lett. 1999, 40, 8693.

[46] Gong, H. G.; Sinisi, R.; Gagne, M. R. J. Am. Chem. Soc. 2007, 129 , 1908.

[47] Gong, H. G.; Gagne, M. R. J. Am. Chem. Soc. 2008, 130, 12177.

[48] Gong, H. G.; Andrews, R. S.; Zuccarello, J. L.; Lee, S. J.; Gagne, M. R. Org. Lett. 2009, 11, 879.

[49] (a) Zhao, C.; Jia, X.; Wang, X.; Gong, H. J. Am. Chem. Soc. 2014, $136,17645$.

(b) Jia, X.; Zhang, X.; Qian, Q.; Gong, H. Chem. Commun. (Camb.) 2015, 51, 10302 .

[50] Zheng, M.; Xue, W.; Xue, T.; Gong, H. Org. Lett. 2016, 18, 6152.

[51] Liu, C. F.; Xiong, D. C.; Ye, X. S. J. Org. Chem. 2014, 79, 4676.

[52] Nicolas, L.; Angibaud, P.; Stansfield, I.; Bonnet, P.; Meerpoel, L.; Reymond, S.; Cossy, J. Angew. Chem. 2012, 124, 11263.

[53] Juhász, Z.; Micskei, K.; Gál, E.; Somsák, L. Tetrahedron Lett. 2007, 48, 7351.

[54] Andrews, R. S.; Becker, J. J.; Gagné, M. R. Angew. Chem. 2010, 122,7432 .

[55] Andrews, R. S.; Becker, J. J.; Gagne, M. R. Org. Lett. 2011, 13 , 2406.

[56] Andrews, R. S.; Becker, J. J.; Gagne, M. R. Angew. Chem., Int. Ed. 2012, 51, 4140 .

[57] Ramnauth, J.; Poulin, O.; Bratovanov, S. S.; Rakhit, S.; Maddaford, S. P. Org. Lett. 2001, 3, 2571. 